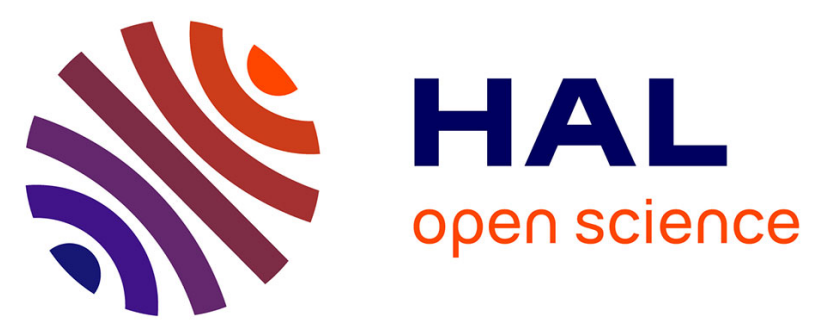

\title{
Groundwater monitoring and characterization by a vertical dipole of superconducting gravimeters in a karst aquifer, France
}

\author{
Sandeep Kumar, Séverine Rosat, Jacques Hinderer, Maxime Mouyen
}

\section{- To cite this version:}

Sandeep Kumar, Séverine Rosat, Jacques Hinderer, Maxime Mouyen. Groundwater monitoring and characterization by a vertical dipole of superconducting gravimeters in a karst aquifer, France. First International Meeting for Applied Geoscience \& Energy Expanded Abstracts, 2021, 10.1190/segam20213582839.1 . hal-03365999

\author{
HAL Id: hal-03365999 \\ https://hal.science/hal-03365999
}

Submitted on 7 Oct 2021

HAL is a multi-disciplinary open access archive for the deposit and dissemination of scientific research documents, whether they are published or not. The documents may come from teaching and research institutions in France or abroad, or from public or private research centers.
L'archive ouverte pluridisciplinaire HAL, est destinée au dépôt et à la diffusion de documents scientifiques de niveau recherche, publiés ou non, émanant des établissements d'enseignement et de recherche français ou étrangers, des laboratoires publics ou privés. 


\section{Groundwater monitoring and characterization by a vertical dipole of superconducting gravimeters in a karst aquifer, France}

Sandeep Kumar*, IIT Roorkee; Severine Rosat and Jacques Hinderer, ITES, University of Strasbourg; Maxime Mouyen, Chalmers University of Technology.

\section{Summary}

The Earth's mass distribution is continuously changing due to physical processes taking place either beneath the subsurface or on the surface. Some of the primary sources for these mass variations are tides in the ocean and solid Earth, atmospheric disturbances and seasonal climate changes. The development of Superconducting Gravimeters (SGs) has made it possible to characterize and monitor such mass variations at micro scales. Our study focuses on the LSBB karst catchment's hydrodynamics using a unique configuration of two SGs located $520 \mathrm{~m}$ depth apart. The installation of a SG (iGrav-31) at the surface of the LSBB several years after the installation of the first (iOSG-24) inside the tunnel has provided several new insights into the understanding of hydrological processes occurring in the LSBB. In this work, we compare differential and residual gravity time-series together with the ERA5 global hydrological loading model. In the subsequent section, we implement a rectangular prism method to compute forward gravity responses using input from the hydrological model. We also numerically evaluate and validate the already published hypotheses (Mouyen et al., 2019) about uncertainties related to groundwater storage's location in this catchment. Based on our observations, we find that most water-storage changes occur in the unsaturated karst zone between both SGs. The misfit between the residual gravity time-series and the local hydrogravity effect computed from ERA5 model shows large lateral fluxes and rapid runoff occurring in the LSBB. Finally, we conclude this work by examining the radial and depth sensitivity of water masses' effect near the SGs, and enlisting some recommendations for further studies.

\section{Introduction}

The importance of characterizing and monitoring the hydrological cycle has rapidly increased due to a large population's growing water demand. The science community is continuously developing more robust monitoring techniques to prevent the depletion of water resources. Karst systems represent an important resource of fresh water but are heterogeneous matrices with fractures and open voids revealing specific hydrodynamic behaviors. Gravimetry has proved to be efficient to provide a direct quantification of the water-storage changes (Creutzfeldt et al., 2014; Hector et al., 2015; Hemmings et al., 2016; Imanishi et al., 2006; Jacob et al., 2008; Pool \& Eychaner, 1995; Van Camp et al., 2006; Wilson et al., 2012). However, by its fundamental integrative properties, gravimetry alone cannot resolve unequivocally the location of the redistribution of water masses. Additional constraints from other measurements or assumptions on the local hydrogeological context would be needed.

Superconducting gravimeters (SGs) are relative instruments that record temporal gravity changes at the Earth's surface and have a wide range of geodynamics applications (Hinderer et al., 2007). In this work we focus on the low-noise underground research laboratory (LSBB) of Rustrel (France), which is a karst hydrosystem where two SGs were installed: an observatory SG, the iOSG-24 which has been recording data since September 2015 (Rosat et al., 2018) and an iGrav instrument (Warburton et al., 2010), the iGrav-31 continuously recording since May 2019. SGs have a precision and a long-term stability appropriate for monitoring water-storage changes (e.g. Hector et al., 2014, Chaffaut et al. 2020).

In this paper we first describe the LSBB site and the unique configuration of two SGs. We then explain the SG data processing used to retrieve gravity residuals that are compared with a global hydrological model. A hydro-gravimetric modeling based on prisms is used to compute hydrological admittances. Then, we use the obtained admittances to compute the gravity effects induced by a water-layer with head changes. From this modeling we finally investigate the gravimetric sensitivity to depth and radius of the water-layer situated in between the two SGs.

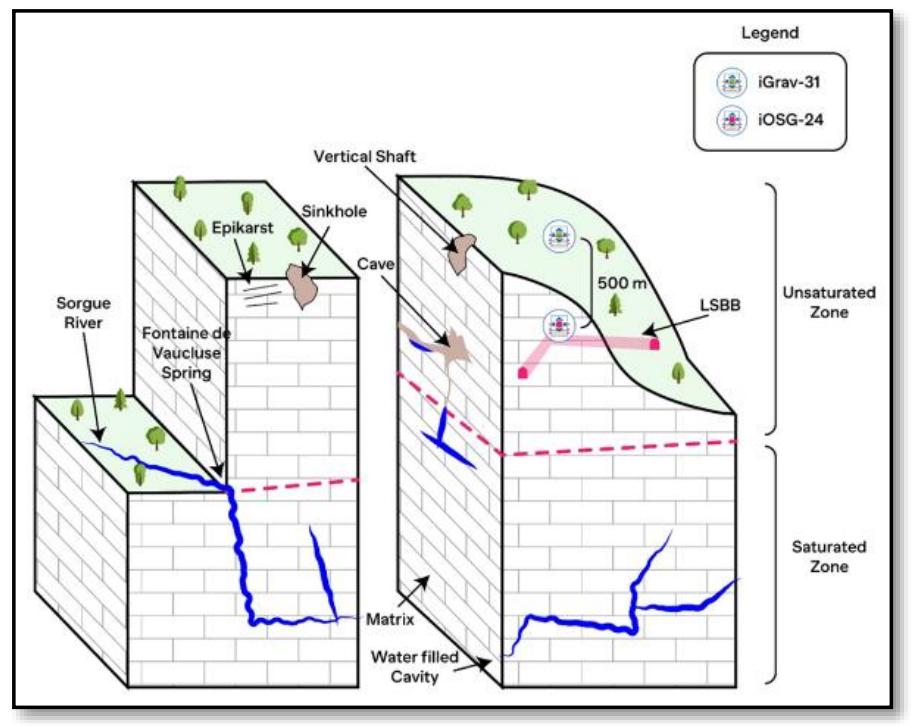

Fig.1: A simplified karst model of the FDV hydrosystem and the LSBB site.

\section{Experimental Site}

Geological and hydrogeological setting

The LSBB site is located in the Fontaine de Vaucluse (FDV) karst hydrosystem in the southeast of France. The catchment area is approximately $1,160 \mathrm{~km}^{2}$, and the average thickness of the unsaturated zone is around $800 \mathrm{~m}$. The FDV lithology mainly consists of $1500 \mathrm{~m}$ thick, massive, and continuous limestone (Masse et al., 1974). The reef limestone part of this catchment may reach a thickness of approximately $450 \mathrm{~m}$. This reef limestone mainly contains Urgonian facies known for high total matrix porosity and covers at least half of the FDV catchment (Masse et al., 1974).

\section{Superconducting Gravimeters setting}

The set-up of both SGs at the LSBB site is such that iGrav-31 is $520 \mathrm{~m}$ nearly vertically above the iOSG-24 (Figure 1). This specific configuration of SGs is unique and should constrain the water-mass location (Mouyen et al., 2019).

\section{Data Processing}

SGs continuously record the time-varying gravity field. Because of the integrative property of gravity, mass redistributions and ground loading from various geophysical sources are recorded. Gravity changes which have the largest amplitudes need to be removed before the data is further assessed for local groundwater monitoring and characterization. Hence, we removed contributions of the solid and oceanic tides using a local tidal model, polar motion effect and atmospheric effects using a local admittance of $-3 \mathrm{~nm} \cdot \mathrm{s}^{-2} \mathrm{hPa}^{-1}$ (e.g. Rosat et al., 2018). Amplitudes of these effects are summarized in Table 1. 
Table 1

\begin{tabular}{|l|l|}
\hline Mass variation factors & Ranges $\left(\mathbf{n m} . \mathbf{s}^{-2}\right)$ \\
\hline Tides & -1900 to 1000 \\
\hline Polar Motion & -10 to 40 \\
\hline Atmospheric Loading & -80 to 50 \\
\hline Non-local Hydrology & -15 to 15 \\
\hline
\end{tabular}

Since we are interested in characterizing the local water storage changes, non-local hydrological effects also need to be corrected for. We use the hydrological loading provided by the EOST loading service (http://loading.ustrasbg.fr/) and calculated for the ERA5 global hydrological model. ERA5 is the fifth generation ECMWF atmospheric reanalysis of the global climate covering the period from January 1950 to the present and is produced by the Copernicus Climate Change Service (C3S). The ERA5 is generated using a more advanced 4D variables assimilation scheme with a sampling interval of 1-hour and horizontal resolution of approximately $30 \mathrm{~km}$. For ERA5, the atmospheric variables are calculated at 139 pressure levels. Residual gravity signal from iGrav31 is strongly correlated with the local ERA5 hydrological loading (Figure 2) while gravity signal from iOSG-24 is anti-correlated. These SG residuals illustrate that water-storage changes at the LSBB site are mostly concentrated in the area below iGrav-31 and above iOSG-24

\section{Methodology}

\section{Gravity Modelling}

In any hydrological system, the water storage change can be inferred from the measured temporal gravity variations. Here, we use a rectangular prism method (prisms of size $1 \mathrm{~m} \times 1 \mathrm{~m} \times 1 \mathrm{~m}$ ) to forward calculate hydrology-induced gravity effect. All three formulas (Forsberg, Macmillan, and point-mass approximations) of this prism method are suitably applied, adhering to radii criteria fixed for a particular survey area (see Leiriao et al., $\underline{2009}$ for details). Neglecting the complexity of the karst medium, we assume that most of the water is uniformly present in carbonate lithology's matrix porosity. We assume a uniform waterlayer of $10-\mathrm{cm}$ thick that follows the topography given by a digital elevation model centered on the SGs location (Figure 3). We then compute the corresponding cumulative gravity effect by offsetting this water layer from the surface (i.e., at iGrav-31 location) toward the saturated zone boundary at approximately $900 \mathrm{~m}$ depth from the surface i.e., $400 \mathrm{~m}$ below the iOSG-24 (Carriere et al., 2016) with $50 \mathrm{~m}$ regular depth-intervals. On further assessing the computed gravity, we find the radius for which water masses are far enough so that gravity change becomes negligible. The obtained hydrological admittances (Figure 4) show that the sensitivity of iOSG-24 to water-storage changes tends to a Bouguer plate with an admittance of $-3.4 \mathrm{~nm} \cdot \mathrm{s}^{-2} \mathrm{~cm}^{-1}$ for radii larger than $4 \mathrm{~km}$, while for iGrav-31 the admittance tends to $3.86 \mathrm{~mm} \cdot \mathrm{s}^{-2} \mathrm{~cm}^{-1}$ for radii larger than $6 \mathrm{~km}$. Please note that for depths of $450 \mathrm{~m}$ and larger, the admittance computed at iOSG-24 location becomes positive since, upon incorporating the topography data at this depth, the water layer is located in average below the SG (Figure 4).

\section{Hydrological Modelling}

We use the ERA5 model to mimic the hydrodynamics of the catchment. The water storage change is estimated by dividing the local ERA5 loading by the infinite Bouguer plate admittance as used in the loading computation (http://loading.u-strasbg.fr/), of $4.2677 \mathrm{~nm} . \mathrm{s}^{-2} \cdot \mathrm{cm}^{-1}$ and multiplied by a $0.25 \%$ specific yield as an estimate of the overall porosity. The hydrological data used here covers the entire catchment (i.e., $1,160 \mathrm{~km}^{2}$ ) which is much larger than the sensitivity zone of both SGs (i.e., 40-60 km² from Figure 4). The simulated gravity response can be computed by multiplying these hydraulic heads obtained from the
ERA5 model with the admittance values computed for various radii and depths as in Figure 4 and divided by $10 \mathrm{~cm}$.

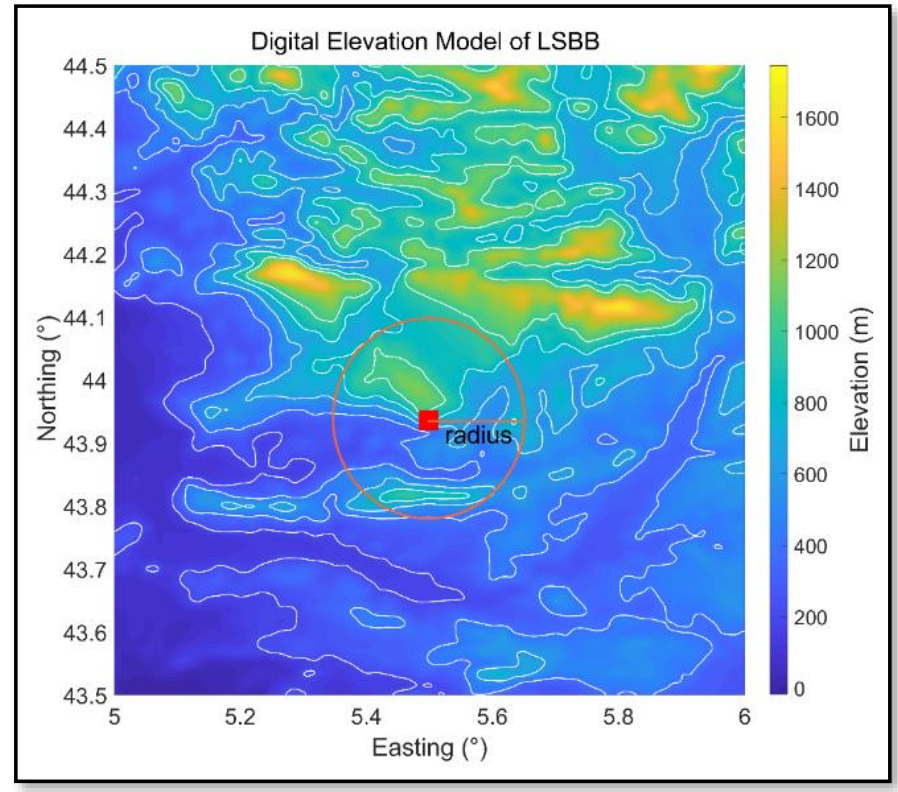

Fig. 3: Digital elevation model of the LSBB catchment centred on iGrav31 (red square). The orange circle illustrates the radius of integration of a hypothetical water layer around iGrav-31.

\section{Results}

The hydrological admittances for iOSG-24 and iGrav-31 and the comparison of SG residual time-series with ERA5 loading imply that most of the groundwater is stored in the zone between both SGs. In the upper unsaturated zone showing the presence of an epi-karst in this catchment is in agreement with the finding by Jacob et al. (2009).

i) We observe that we cannot constrain both depth and extent of the water layer simultaneously.

ii) At a particular depth between both SGs (i.e., here $\mathrm{d}=300 \mathrm{~m}$ ), we see that the modelled gravity effect corresponding respectively to an extent of $900 \mathrm{~m}$ (iGrav-31) or $40 \mathrm{~m}$ (iOSG24 ) best fit the gravity residuals (Figure 5, a, and b).

\section{Discussions}

We observe that the ERA5 loading model shows a few trends of both overestimation and underestimation of gravity effects (Figure 2, a) compared to residual gravity time series recorded by the iGrav-31. Several plausible reasons can be outlined:

i) The excessive lateral fluxes of groundwater may be inhibiting the vertical infiltration of rainwater into the ground.

ii) The SGs are located on the boundary of the catchment, and hence, the water mass distributions outside it may be altering gravity.

iii) The time lags between residual gravity and the hydrological model may be the cause for this misfit.

For a water layer of a few kilometer radial extent, the effect of depth on gravity becomes negligible. Therefore, in that case, finding the depth of storage becomes very uncertain.

\section{Conclusions}

The residual and differential gravity time series of the SG vertical dipole clearly show groundwater redistribution's seasonal effects at LSBB. The simulated gravity responses from ERA5 atmospheric model indicate that most groundwater is stored in the zone between both SGs. The fitting of 
the observed gravity changes with prism-modelled gravity effects has helped us to delineate the boundary of the stored groundwater in this catchment. However, the misfit seen in the individual fitting of the modelled gravity to each SG gravity residual show that further work is needed to improve the simplified hydro-gravimetric model proposed in this study.

\section{Acknowledgments}

The iOSG-24was funded by the EQUIPEX MIGA and by the European FEDER 2006-2013. The iGrav-31 was funded by the EQUIPEX CRITEX.

(a)
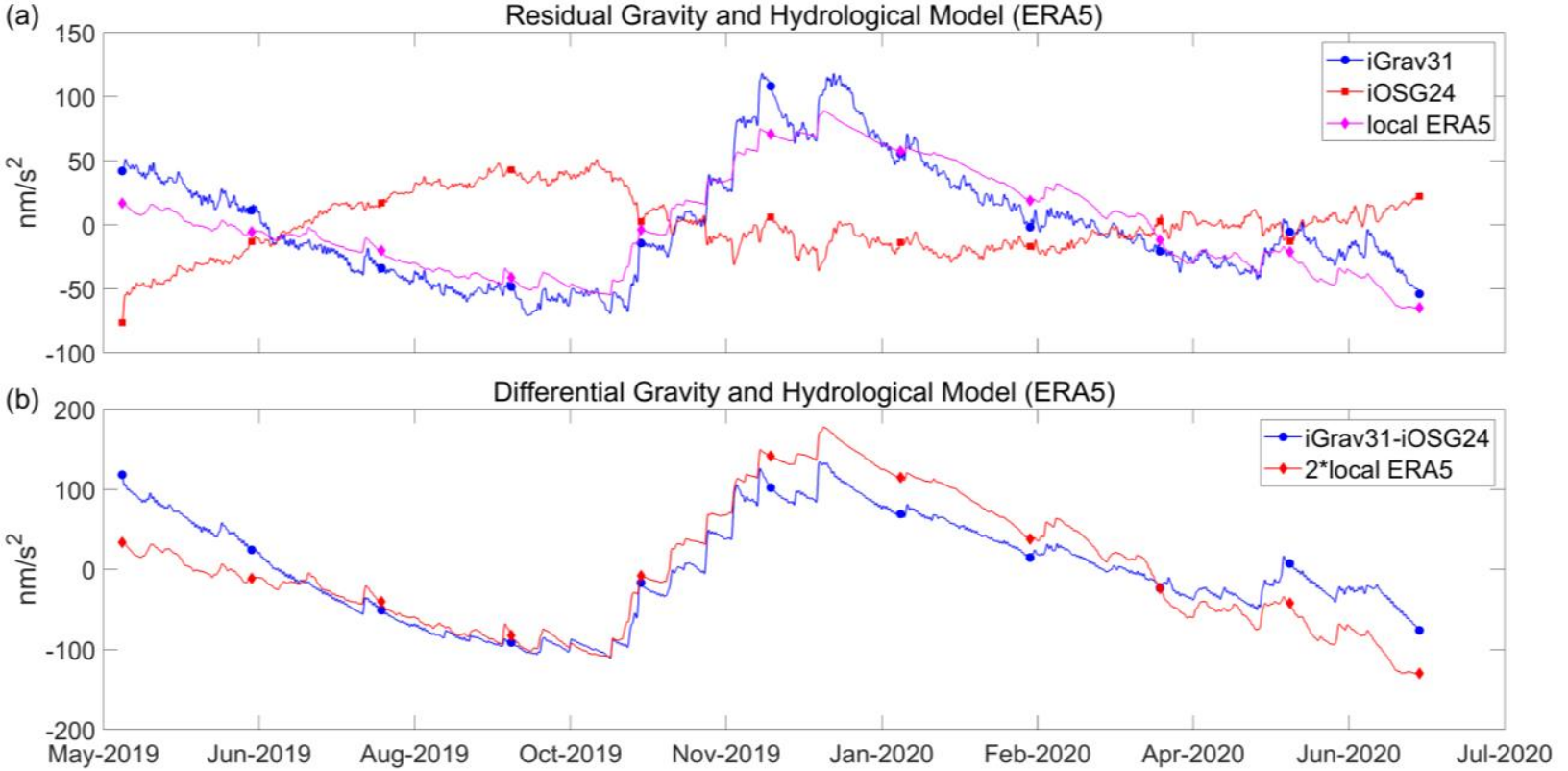

Fig. 2: a) Residual gravity time-series recorded by iGrav-31 (blue line) and by iOSG-24 (red line) compared to the local hydro-gravity loading computed from ERA5 hydrological model (magenta line); b) Differential gravity signal (blue line) compared to the differential local hydro-gravity effect from ERA5 hydrological model.

(a)

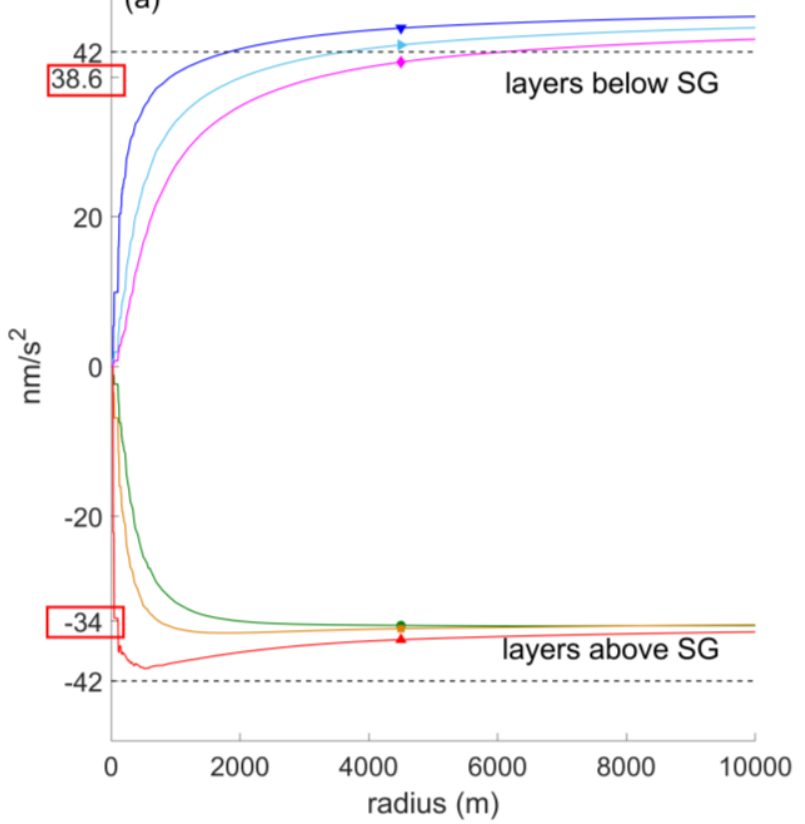

iGrav-31

(b)

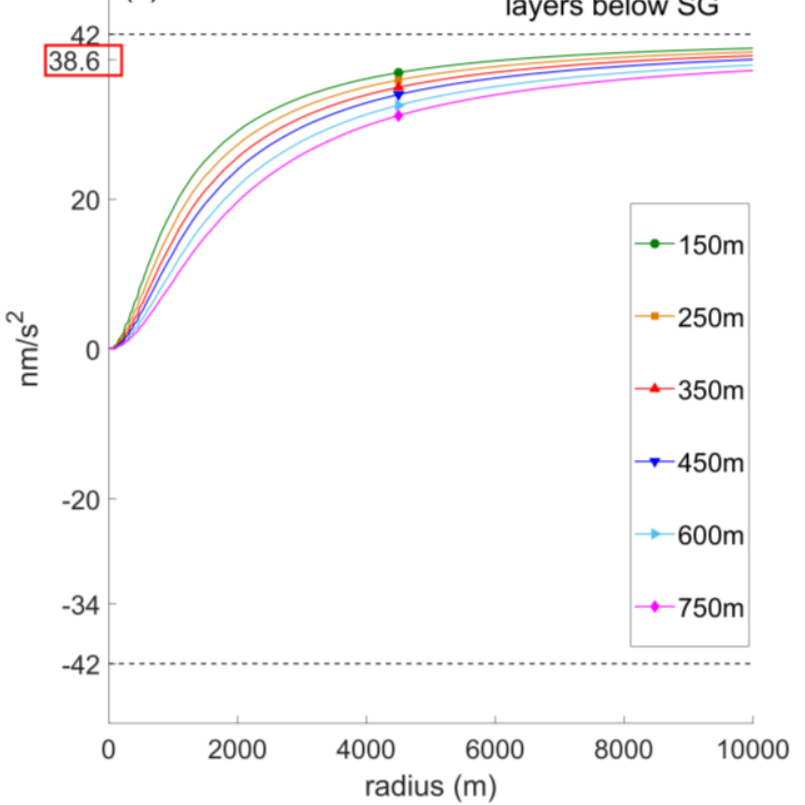

Fig. 4: Gravity effects of the 10-cm water layer as a function of its radius and its depth. Red boxes mark the average gravity effect for layers above/below the SGs. 

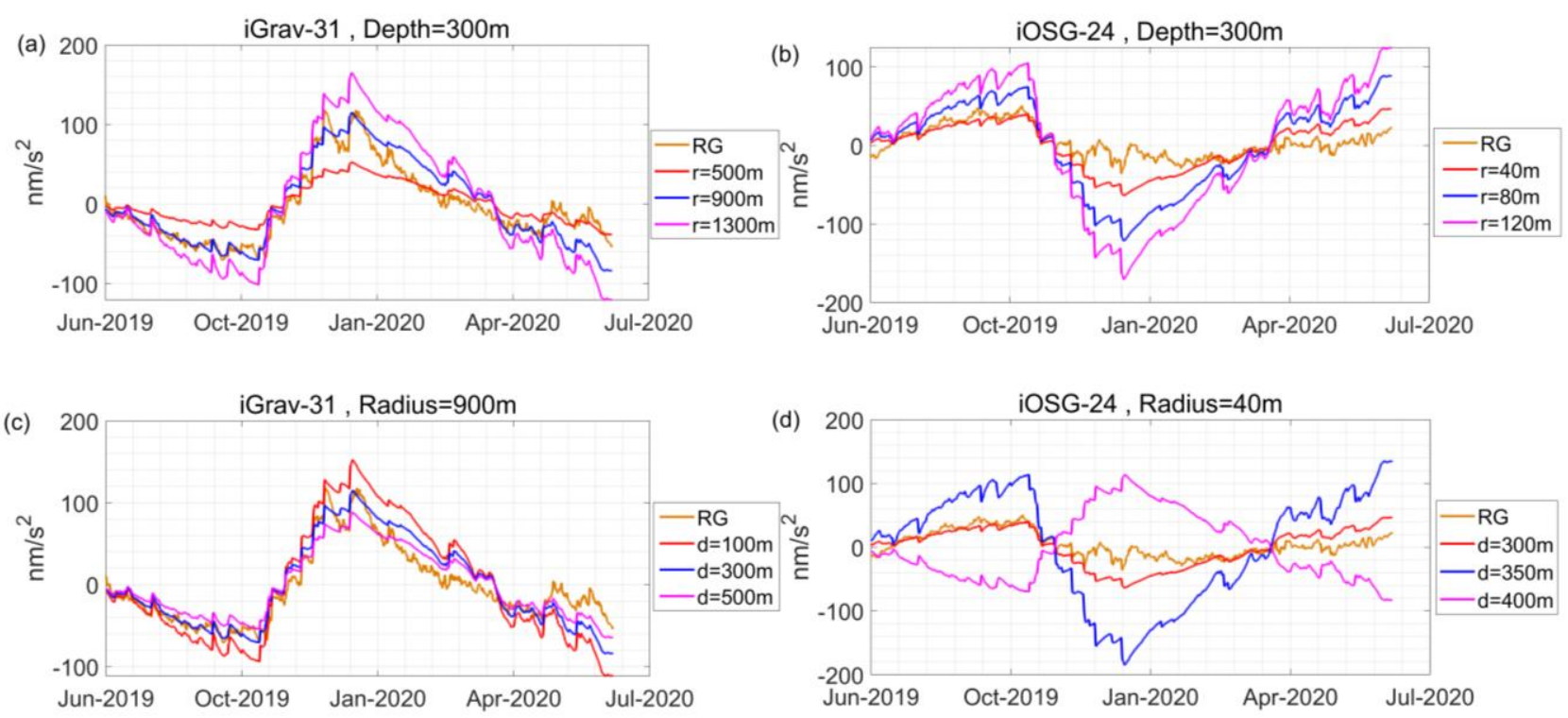

Fig. 5: Residual gravity (RG) time-series recorded by a) iGrav-31 and b) iOSG-24 compared to the prism modelled gravity at a depth of $300 \mathrm{~m}$ from the surface for various radii of the water-layer; Residual gravity (RG) time-series recorded by a) iGrav-31 and b) iOSG-24 compared to the prism modelled gravity at radius of $900 \mathrm{~m}$ and $40 \mathrm{~m}$ from the SGs location respectively, for various depths of the water-layer. 